\title{
Patient-Specific Modelling in Orthopedics: From Image to Surgery
}

\author{
G.T. Gomes ${ }^{1}$, S. Van Cauter², M. De Beule ${ }^{2}$, L. Vigneron ${ }^{3}$, C. Pattyn ${ }^{1}$, E.A. \\ Audenaert $^{1^{*}}$ \\ ${ }^{1}$ Ghent University Hospital, Ghent, Belgium, Emmanuel.Audenaert@UGent.be \\ ${ }^{2}$ IBiTech-bioMMeda, Ghent University, Ghent, Belgium \\ ${ }^{3}$ Orthopedic Department, Materialise NV, Leuven, Belgium \\ * Corresponding author
}

\begin{abstract}
In orthopedic surgery, to decide upon intervention and how it can be optimized, surgeons usually rely on subjective analysis of medical images of the patient, obtained from computed tomography, magnetic resonance imaging, ultrasound or other techniques. Recent advancements in computational performance, image analysis and in silico modeling techniques have started to revolutionize clinical practice through the development of quantitative tools, including patient-specific models aiming at improving clinical diagnosis and surgical treatment. Anatomical and surgical landmarks as well as features extraction can be automated allowing for the creation of general or patient-specific models based on statistical shape models. Preoperative virtual planning and rapid prototyping tools allow the implementation of customized surgical solutions in real clinical environments. In the present chapter we discuss the applications of some of these techniques in orthopedics and present new computer-aided tools that can take us from image analysis to customized surgical treatment.
\end{abstract}

Keywords: Musculoskeletal modelling, patient-specific models, surgical planning. 


\section{Virtual anatomical landmark extraction}

\subsection{Anatomical landmarks in orthopedics}

The identification of reference parameters or anatomical landmarks is a wellestablished technique in orthopedic surgery. Anatomical features are used for various applications. Many morphological parameters (e.g. distances, angles) are quantified based on landmarks (Paley 2002). These measurements can serve as a guideline for distinguishing dysplastic from normal morphologies (Delaunay et al. 1997). Also, many studies have shown that accurate prosthetic component positioning is a key factor for the success of joint replacement surgery and have presented recommendations for the orientation angles (Yoon et al. 2008). Joint kinematics is often described by the relative motion of joint coordinate systems that are attached to the bones. These joint coordinate systems can be defined based on anatomical features (Grood and Suntay 1983). Moreover, surgical navigation systems rely on landmarks. Image-based navigation requires patient-to-image registration and this process often relies on registration points that are determined on the image and have to be recognized during the operation (Nizard 2002). Imagefree navigation systems use landmarks to create anatomical reference frames that relate the position and orientation of the reference frames that are attached to the patient's bones, to the underlying bony anatomy (Siston et al. 2007). Finally, different landmarks can be used to determine the insertion locations in ligament reconstruction (Schottle et al. 2007, Ziegler et al. 2010). Anatomical features have thus proven to be applicable throughout all steps of the patient treatment process: diagnosis, preoperative planning, intraoperative navigation and postoperative follow-up.

Anatomical landmarks can be quantified in different ways. On live subjects, they can be located using manual palpation and digitized using a probe. In addition, surgical navigation systems allow to determine landmarks by means of a kinematic analysis of the patient's joint (e.g. centre of the hip, knee and ankle). Software programs are available for manual identification of landmarks on digital medical images and three-dimensional (3D) computer models. Moreover, various techniques for automatic landmark extraction are being developed. An important factor in landmark-based applications is the use of standardized definitions to allow for better result comparison and data exchange (Van Sint Jan and Della Croce 2005, Van Sint Jan 2007).

\subsection{Virtual landmark identification}

Several imaging methods allow users to virtually quantify landmarks on the inner structures of the body. 2D radiography is the most common and widely avail- 
able imaging technique in orthopedics. However, radiographic images are characterised by the superimposition of anatomical structures and by magnification and distortion errors. Computed tomography (CT) and magnetic resonance (MR) imaging have been used to obtain more detailed, cross-sectional images. Furthermore, multiplanar reformatted images and 3D surface renderings can be generated from these 2D slices.

Most orthopedic systems that use digital images or models require the physician to manually mark the landmarks, relying on his good judgement and experience. Therefore, intra- and interobserver variability is an important factor that should be taken into account when using landmark-based clinical parameters. Several studies have been performed to assess the reproducibility of landmark identification on CT or MR (-based) images and the corresponding morphological parameters (Cerveri et al. 2010, Lerner et al. 2003, Nofrini et al. 2004, Subburaj et al. 2009, Victor et al. 2009, Yoshino et al. 2001), see Table 1.

Table 1 Variability of manual landmark identification. Images: scout view (SV), slices (SL), multiplanar reformatted (MR), 3D model (MO). Variability definition using values from several analyses: difference from the mean value (DFM), standard deviation (SD), pairwise difference (PD), standard error of the measurement (SEM). Averaged over: coordinate axes (CA), analyses (AN), datasets (DS), parameters (PA).

\begin{tabular}{|c|c|c|c|c|c|c|c|}
\hline Reference & $\begin{array}{l}\text { \# data } \\
\text { sets }\end{array}$ & $\begin{array}{c}\text { \# parame } \\
\text { ters }\end{array}$ & Images & $\begin{array}{l}\text { Variability } \\
\text { definition }\end{array}$ & $\begin{array}{l}\text { Averaged } \\
\text { over }\end{array}$ & Observer & Value \\
\hline $\begin{array}{l}\text { Victor et } \\
\text { al. } 2009\end{array}$ & 6 & 27 & MO & DFM & AN \& DS & inter & $\begin{array}{l}0.4-1.4 \mathrm{~mm} \\
0.05-1.44^{\circ} \\
0.3-3.5 \mathrm{~mm} \\
0.08-3.16^{\circ}\end{array}$ \\
\hline $\begin{array}{l}\text { Cerveri et } \\
\text { al. } 2010\end{array}$ & 20 & 7 & MR & DFM & $\mathrm{AN}$ & inter & $\begin{array}{l}\leq 3.0 \pm 2.1 \mathrm{~mm} \\
\leq 3.5 \pm 2.2^{\circ} \\
\leq 3.1 \pm 2.0 \mathrm{~mm} \\
\leq 3.7 \pm 2.3^{\circ}\end{array}$ \\
\hline $\begin{array}{l}\text { Subburaj } \\
\text { et al. } \\
2009\end{array}$ & 3 & 14 & MO & SD & $\begin{array}{l}\text { CA, AN \& } \\
\text { DS }\end{array}$ & inter & $2.15-5.98 \mathrm{~mm}$ \\
\hline $\begin{array}{l}\text { Yoshino } \\
\text { et al. } \\
2001\end{array}$ & $33 / 96$ & 2 & SL (axial) & PD & DS & Inter & $\begin{array}{l}0.4 \pm 0.4^{\circ}-1.1 \pm \\
0.6^{\circ}\end{array}$ \\
\hline $\begin{array}{l}\text { Nofrini et } \\
\text { al. } 2004\end{array}$ & 4 & 4 & $\begin{array}{l}\text { SV (frontal } \\
\text { and lateral) } \\
\& \text { SL (axial) }\end{array}$ & PD & AN \& DS & $\begin{array}{l}\text { intra } \\
\text { inter }\end{array}$ & $\begin{array}{l}2.8 \pm 1.8-5.4 \pm 2.7 \\
\mathrm{~mm} \\
3.1 \pm 1.6-5.4 \pm 2.7 \\
\mathrm{~mm}\end{array}$ \\
\hline $\begin{array}{l}\text { Lerner et } \\
\text { al. } 2003\end{array}$ & 7 & 9 & $\begin{array}{c}\text { SL (axial } \\
\text { and sagittal) }\end{array}$ & SEM & $\begin{array}{l}\text { CA, AN } \\
\text { DS \& PA }\end{array}$ & intra & $0.41 \mathrm{~mm}$ \\
\hline
\end{tabular}


A direct comparison between studies is not feasible, because of the different methods that are used, but their results indicate that mean variabilities of 2-3 mm/ are not uncommon for some of the landmarks and parameters.

In addition, models have been developed to determine the deviation of clinical parameters that arises from landmark variability. Morton et al. 2007 found a significant variability of absolute kinematic parameters of up to $6.5^{\circ}$ and $4.4 \mathrm{~mm}$ for tibiofemoral and $7.6^{\circ}$ and $6.5 \mathrm{~mm}$ for patellofemoral kinematics, assuming standard deviations of up to $2 \mathrm{~mm}$ for the anatomical landmark locations. The simulation results of Wolf et al. 2005 indicate that version and abduction errors in acetabular cup alignment can be as large as $\pm 10^{\circ}$ and $\pm 6^{\circ}$, respectively, assuming a $4 \mathrm{~mm}$ error in anterior pelvic plane landmark localization. Although these studies used rather high variability for all landmarks involved, they illustrate the significance of estimating the resulting errors in the clinical parameters to preclude misinterpretation of data.

\subsection{Automatic landmark extraction}

Manual localisation of landmarks is inherently characterized by inter- and intraobserver variability. Although computer methods have been developed to assist the user in landmark identification (Hankemeier et al. 2006), the procedure remains time-consuming and a high level of expertise and alertness is required to obtain precise measurements. To overcome these drawbacks, automated landmark extraction techniques are increasingly being used (Ehrhardt et al. 2004, Subburaj et al. 2010). Their validity is usually tested by comparing the parameters obtained by the automated methods to a reference parameter set obtained by manual expert analysis.

We have developed a heuristically driven approach for fast automated landmarks, regions and other parameters extraction (Gomes 2011). Applied to the human femur, it extracts the following parameters: epycondyles (most lateral and medial), transepicondylar axis, condyles (most inferior and posterior locations), intercondylar fossa, femoral condylar axis, centre and radius of the femoral head, greater trochanter (most lateral and most superior locations), lesser trochanter, quadrate tubercle, iliofemoral ligament insertions, trochanteric fossa, linea aspera, abductor tubercle, anterior and posterior cruciate ligament insertions, axis of the neck, isthmus of the neck, optimum antegrade and retrograde nail insertions from least squares B-spline fit to the shaft medial points or from least squares elliptic hyperboloid fitting to the shaft and Bezier curve computation from the hyperboloid's medial axes (Gomes 2011, Van Cauter et al. 2011), inclination and version of the head and bending between distal and proximal parts of the shaft (Figs. 1 and 
2). A high resolution bone reconstructed from sub millimetre CT voxels (with over 50,000 vertices), can be fully analysed in less than $10 \mathrm{~s}$. on an average desktop machine running Matlab. The algorithms have been tested using a database of 100 CT scanned femurs with wide normal and some pathological variability.

These automated techniques offer several advantages over manual analyses. They may save time for the physician and allow rapid data processing to obtain morphological measurements for large control and patient populations. Moreover, they eliminate observer variability by using standardized procedures, which may result in an improved (e.g. pre- and postoperative) data comparison and exchange. Their validity and robustness should always be tested using large datasets. It should also be taken into account that standardized algorithms may not always be able to correctly capture the features in severely deformed anatomies but this can be addressed with the use of shape models that capture such variability.

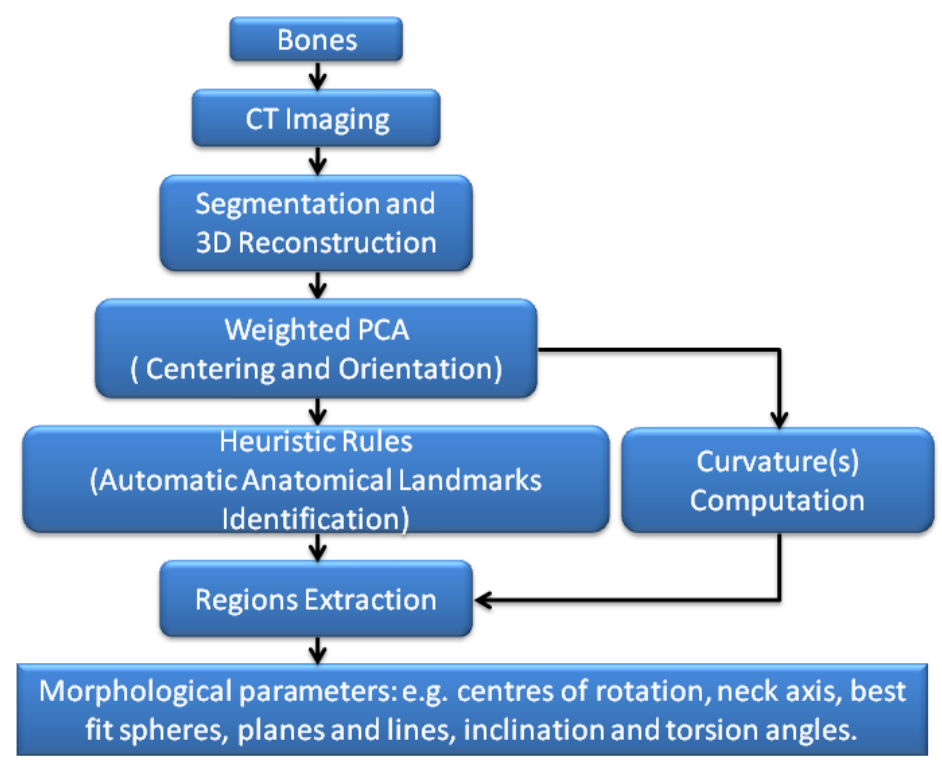

Fig. 1 Overview of the methodology for landmarks and other features extraction. 


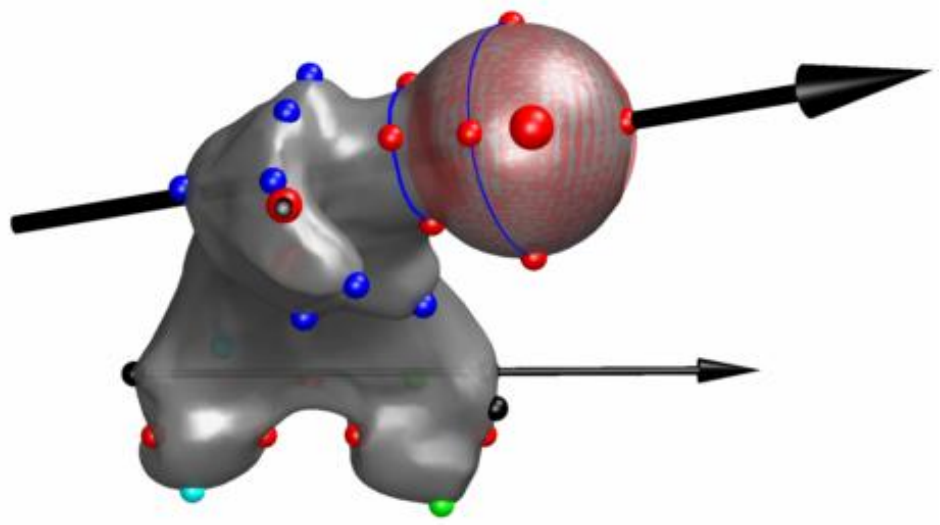

(a)

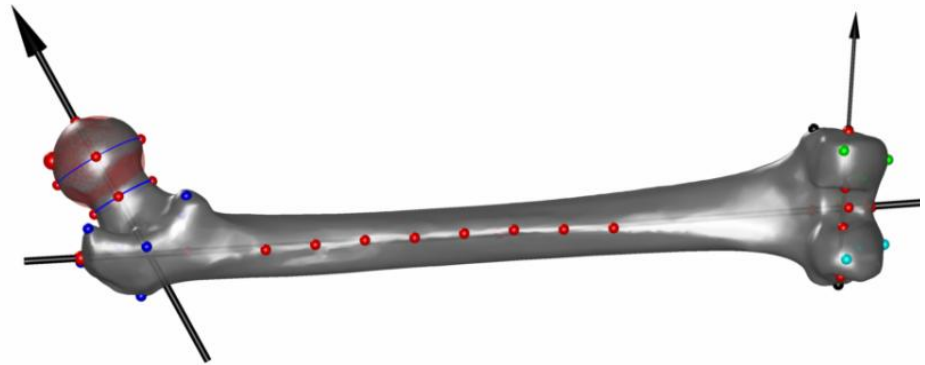

(b)

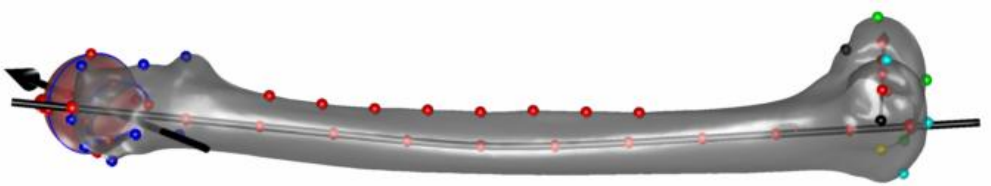

(c)

Fig. 2 Superior (a), posterior (b) and lateral (c) views of a left side femur with extracted features i.e. landmarks, axes, best fits (Gomes 2011).

\section{Statistical shape models}

In medical imaging, statistical shape analysis provides an important and increasingly popular means for computing 3D models of the patient's anatomy. This capability has been used for:

- 3D reconstruction of anatomic structures from sparse patient-specific input data (Barratt et al. 2008, Benameur et al. 2003, Blanz et al. 2004, Fleute et al. 1998, Kurazume et al. 2009, Rajamani et al. 2007, Stindel et al. 2002, Zheng et al. 2008, Zheng and Schumann 2009). While 3D image datasets (e.g. CT, MR) can 
be acquired for segmentation purposes, the constraints of clinical reality (e.g. time, cost and exposure to unacceptable levels of ionizing radiation) preclude their widespread use, on the other hand 2D medical imaging modalities such as $\mathrm{X}$-rays, ultrasound or fluoroscopy, or direct pointer digitization can be used to derive patient-specific information pre- or intraoperatively from the patient's anatomy for the purpose of 3D reconstruction. The acquired patient-specific data can be processed and used to deform a shape model to finally reconstruct the patient's anatomy

- Predicting the shape of one bone from the observation of another from the same joint (Yang et al. 2008)

- Segmentation (Baldwin et al. 2010, Behiels et al. 2002, Heimann et al. 2009, Tang and Ellis 2005), SSM allows encapsulation of prior anatomical knowledge for compensating low contrast and/or high levels of noise in the images, such models can achieve robust segmentation by constraining the possible shapes (Cootes and Taylor 2004, Cootes et al. 1995)

- Design of prosthesis and biomechanical finite element analysis (Bryan et al. 2009)

- Aiding in the detection of pathologies related to shape (e.g. cam impingement, osteoarthritis), anatomical differences related to sex and aging (Styner et al. 2005).

\subsection{Basic concepts}

Statistical shape models (SSM) aim at describing the natural variability of a shape, e.g. the morphological variation of the same bone from different subjects or the organ of one subject through time. The general idea behind SSM is to perform a linear decomposition of the shape variability from a set of training data by defining a mean shape and modes of deformations under some mathematical criteria. The most commonly used principal component analysis (PCA) estimates orthogonal directions which maximize the variance. Other linear decompositions can be used, based on principal factor analysis or independent component analysis. PCA is mathematically defined as an orthogonal linear transformation that transforms the data to a new coordinate system such that the greatest variance by any projection of the data comes to lie on the first axis (called the first principal component), the second greatest variance on the second axis, and the third greatest variance on the third axis and so on. PCA is theoretically the optimum transform for a given data in the least squares sense, its operation can be thought of as revealing the internal structure of the data in a way that best explains the variance in the data (Dryden et al. 1998, Jolliffe 2002). PCA-based classification methods implicitly assume that the data was generated by a Gaussian probability distribution and is therefore less than optimal if this assumption is not valid.

Shape descriptors used in medical image analysis include both parametric models, such as Fourier descriptors or spherical harmonic functions, and non- 
parametric models such as landmark-based descriptors, deformation fields, distance transforms and medial axes. We explore landmark-based descriptors or more specifically shape analysis based on densely sampled 3D point distribution models. Landmarks can be generally grouped as:

Anatomical landmarks: Points assigned by an expert or automatically extracted that have a correspondence between organisms in some biologically meaningful way.

Mathematical landmarks: Points located on an object according to some mathematical or geometrical property, e.g. centre of a ball and socket joint obtained through least squares fitting of a sphere.

Pseudo-landmarks: Constructed points on an object either around the outline or between anatomical landmarks.

In general, a statistical shape model is built from a training set of shapes $\left\{\right.$ Shape $\left._{\mathrm{i}}: i=1, \ldots, n_{s}\right\}$ where $n_{s}$ is the total number of shapes. Each shape can be approximated by $n_{p}$ points sampled on its $3 \mathrm{D}$ surface such that the individual points are placed in corresponding positions across the set of shapes. This results in a set of initial shape vectors $\left\{S_{i}: i=1, \ldots, n_{s}\right\}$ which form a representation of the whole set of shapes in a common shape space $\mathbb{R}^{3 \times n_{p}}$.

Using PCA, each shape vector can be expressed using a linear model (eq. 1), that is, new shape instances can be obtained by adding a linear combination of the principal shape modes to the mean surface:

$S=\bar{S}+P b=\bar{S}+\sum_{i=1}^{n_{s}-1} p_{i} b_{i}$

where $\bar{S}=\frac{1}{n_{s}} \sum_{i=1}^{n_{s}} S_{i}$ is the mean shape vector and $P=\left\{p_{i}\right\}$ the matrix of eigenvectors of the covariance matrix. The corresponding eigenvalues $\left\{\lambda_{i}\right\}$ describe the amount of variance in the direction of the eigenvectors, in practice, what is retained is not simply the directions corresponding to non-zero eigenvalues, but instead that ordered set, from highest to lowest, which adds to a certain amount of the total variance of the data, where the total variance of the data is given by $\sum_{i=1}^{n_{s}-1} \lambda_{i}$, and the variance up to $n_{m}$ is $\sum_{i=1}^{n_{m}} \lambda_{i}$, here the number of modes $n_{m}$ is chosen to be the lowest value such that the cumulative variance up to $n_{m}$ is above a certain threshold (e.g 99\%). This is a somewhat arbitrary value allowing for a compact model whilst capturing most of the variance in the data. Other commonly used values are 90 and $95 \%$. The shape parameters $b=\left\{b_{i}\right\}$ control the contribution of modes of variation, $b_{i}$ is usually chosen to be bounded between $-3 \sqrt{\lambda_{i}}$ and $+3 \sqrt{\lambda_{i}}$. 
In order to obtain a correct statistical model all points on each surface must correspond in an anatomical meaningful way, and their coordinates must be given relative to a common frame of reference (alignment). These two steps are fundamental, since incorrect correspondences can either introduce too much variation or lead to erroneous instances of the model (Dryden et al. 1998, Lamecker et al. 2004).

\subsection{Alignment and non-rigid registration}

Before establishing correspondences using non-rigid surface registration, alignment and scaling can be achieved using the automatically extracted anatomical landmark points and an iterative approach using Procrustes analysis (Bookstein 1997, Cootes et al. 2004).

The non-rigid registration of the bones is based on the method introduced by Rueckert et al. 1999. The key idea of this method is to embed a surface in a volumetric mesh which defines the continuous deformation field. The mesh is subsequently subdivided into higher resolution levels by inserting control points into the current level of control points and decreasing the mesh space. The deformation is affected by the spacing of the control points: a larger spacing leads to a smoother global shape while a relatively smaller spacing leads to higher local deformation. It is based on a Free Form Deformation (FFD) with a B-spline grid, and error calculation is done with normalized mutual information. To define a spline based FFD, we denote the domain of the bone volume as $\Omega=\{(\mathrm{x} ; \mathrm{y} ; \mathrm{z}) \mid 0 \leq \mathrm{x}<\mathrm{X}, 0 \leq \mathrm{y}$ $<\mathrm{Y}, 0 \leq \mathrm{Z}<\mathrm{Z}\}$.

Let $\Phi$ denote a $n x \times n y \times n z$ mesh of control points $\Phi(i, j, k)$ with uniform spacing $d x, d y$ and $d z$. Then, the FFD with transformation $\mathrm{T}$ can be written as the 3-D tensor product of the 1D cubic B-splines (eqs 2-4)

$T=\sum_{l=0}^{3} \sum_{m=0}^{3} \sum_{n=0}^{3} B_{l}(u) B_{m}(v) B_{n}(w) \Phi(i+l, j+m, k+n)$

where

$i=[x / n x]-1, \quad j=[y / n y]-1, \quad k=[z / n z]-1$,

$u=x / d x-[x / d x], v=y / d y-[y / d y], \quad w=z / d z-[z / d z]$

$\boldsymbol{B}_{\boldsymbol{l}}$ represents the $l$ th basis function of the B-spline 


$$
\begin{aligned}
& B_{0}(u)=(1-u)^{3} / 6 \\
& B_{1}(u)=\left(3 u^{3}-6 u^{2}+4\right) / 6 \\
& B_{2}(u)=\left(-3 u^{3}-3 u^{2}+3 u+1\right) / 6 \\
& B_{3}(u)=u^{3} / 6
\end{aligned}
$$

\subsection{Illustrative results: femur bone main modes of shape variation}

Compactness of an SSM of the femur, based on 43 femurs (Fig. 3). The first mode explains approximately $48 \%$ of the total observed shape variance, the seven first combined modes account for about $90 \%$ and the 20 first modes capture more than $99 \%$ of shape variation.

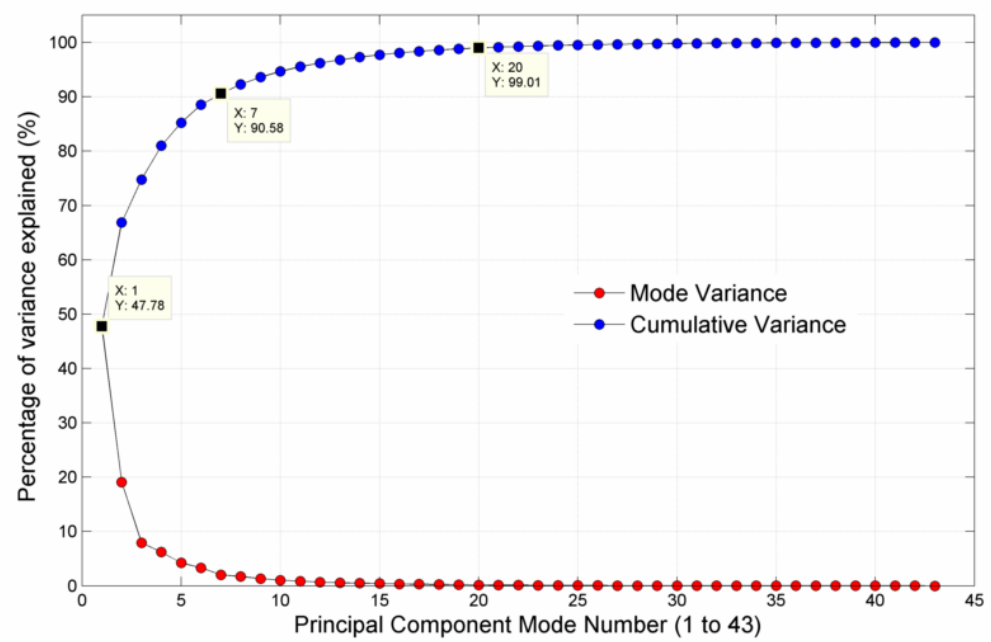

Fig. 3 Compactness of an SSM of the femur, based on 43 bones.

In Fig. 4, the first 3 modes of shape variance and mean shape can be visualized. 

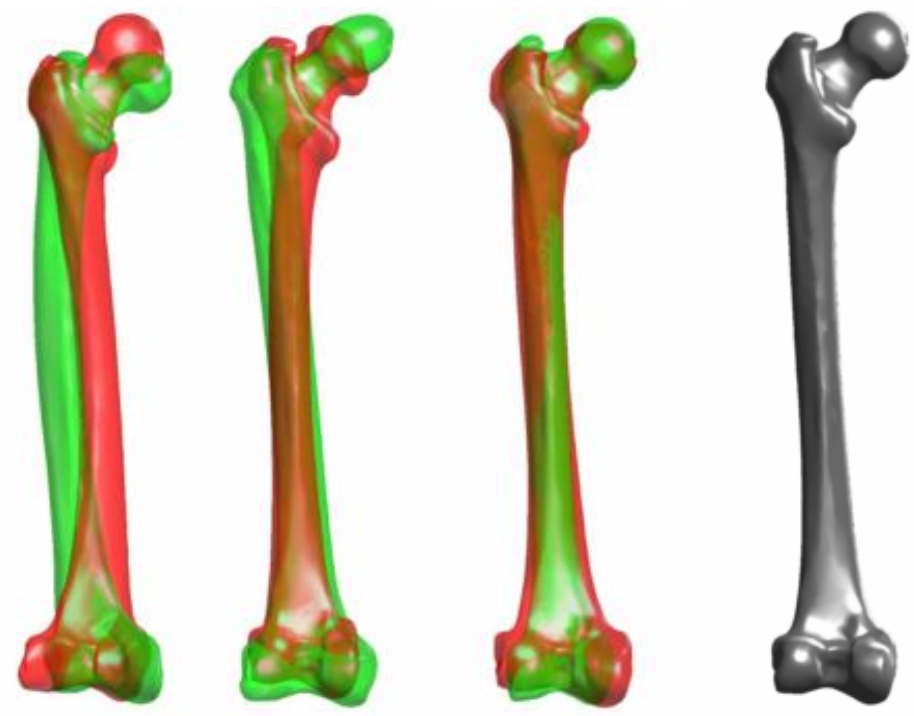

Fig. 4 Mean shape and variations of the shape of the full femur for the first 3 modes, based on an SSM built from 43 training femurs. From left to right modes 1 to 3 and mean shape (grey), green using a shape parameter of $-3 \sqrt{2_{\mathrm{i}}}$ and red $+3 \sqrt{2_{\mathrm{i}}}$.

\section{Integration of virtual planning into computer-aided surgery}

Diagnosis and correction of osseous deformity and soft tissue function are among the most common aspects of orthopedic surgery. Commercial and opensource computer aided-tools aiming at virtual analysis and simulation have increasingly began to emerge parallel to the technical improvements in imaging techniques, allowing for detailed volumetric visualization of the musculoskeletal system (Blemker et al. 2007, Delp et al. 2007, Delp and Loan 1995). Techniques such as CT and MR imaging are currently routinely available. The goals of computer-based surgery simulation are to enable the surgeon to experiment with different surgical procedures (osteotomies, grafts, implants, etc) in an artificial environment and to try predicting the outcome of an intervention before the actual surgery. However, the final surgical outcome and accuracy in implementing the virtual plan are related to the surgeon's ability to reproduce the planned surgery.

One of today's state of the art examples of simulation software is OpenSim, an open-source software system developed at Stanford University that lets users create and analyze dynamic simulations of movement (Delp et al. 2007). It can be used to study common orthopedic surgical techniques such as muscle transfers, and allows for analysis of how muscle moment arm, optimal muscle fiber length, and tendon slack length influence the variation of muscle force with respect to 
changes in joint angle. Customized models can be created by scaling of a generic model, (Fig. 5).

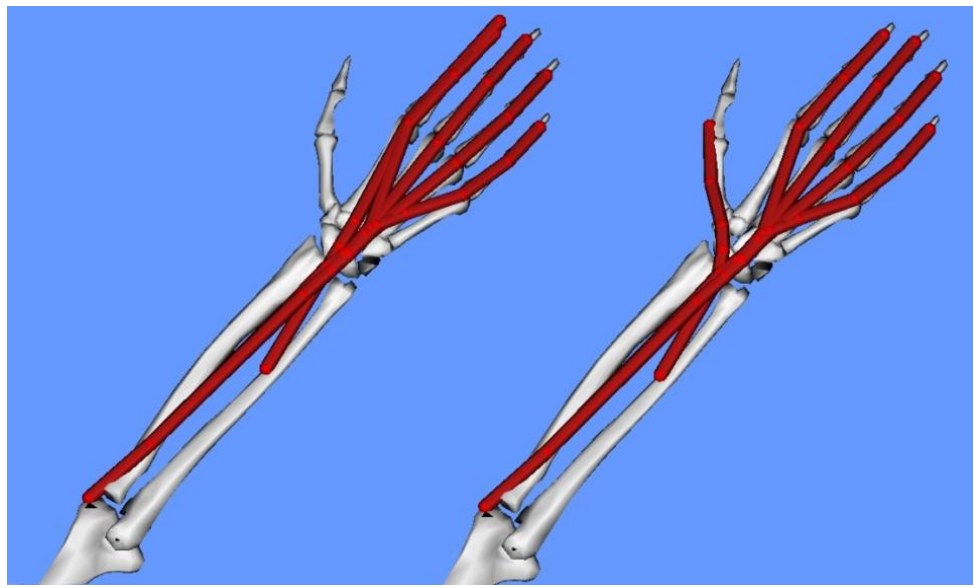

Fig. 5 OpenSim ${ }^{\circledR}$ wrist model simulating an extensor indices proporius to extensor pollicus longus transfer.

Despite the growing dissemination and technical excellence of these virtual modeling tools, implementation of a virtual plan in the operating theatre and the linkage to the available navigational systems remain a restraint to full computer aided assistance, from image to actual surgery. Part of the problem lies in the fact that both academic researchers and industry, each in their individual attempt to attain international excellence, have been focusing on and specializing in subdisciplines such as image analysis, modeling, surgical robotics or navigation with limited integration possibilities between each.

Currently, "integrated and multiscale approaches" have become important research goals in ICT for health. A promising example is the recently granted European Project Tlemsafe, joining academic experts in image analysis and segmentation, musculoskeletal modeling and navigation technology, and including industrial partners such as Materialise Inc (Image analysis and rapid prototyping), Anybody Inc (Muscoloskeletal modeling) and Brainlab Inc (surgical navigation).

To date, a few methods are already readily available to make the integration from image over virtual planning to computer-assisted surgery. We present two such solutions, either using customized surgical guides or by generating a virtual plan in DICOM format that can be read by common navigation systems. 


\subsection{Patient-specific surgical guides}

In the orthopedic field, surgical standard instruments have been used for years to transfer the planning into surgical gestures. However, the exact position of standard instruments relative to patient anatomy was not defined, and had to be adapted intraoperatively in order to reproduce the planning as well as possible. As opposed to standard instruments, patient-specific guides, or templates/jigs, started to be developed in the late nineties (Birnbaum et al. 2001, Radermacher et al. 1998). These surgical guides generally possess a surface that exactly fits a patient's anatomical surface, e.g. bone or cartilage surface. During surgery, the guide is positioned onto this anatomical surface and its position tested until the optimal match between guide and patient anatomical surface is found, which indicates that the guide is correctly positioned thanks to a unique fitting position.

While standard instruments are built using classical manufacturing techniques, e.g. injection molding, the use of similar techniques for building patient-specific guides would be extremely cost-inefficient. This explains why the first patientspecific guides were built using a milling technique, starting from a standardized block that was progressively milled to reach the final shape of the surgical guide (Redermacher et al. 1998). An interesting feature of this technique is the existence of desktop milling machines. The design and production of these guides were thus presented as a future integrated process in the medical routine and performed by the medical staff.

Another means for building patient-specific guides are the rapid prototyping, or additive manufacturing, techniques (Hopkinson et al. 2005). The basic principles of these techniques consist of, starting from a virtual object to be produced, virtually slicing it into a stack of layers. The object is then built layer per layer. Different materials can be used for rapid prototyping objects, and in case of surgical guides, the selective laser sintering technique based on polyamide powder is often used, mainly because of its manufacturing accuracy, biocompatibility, and good response to heating for sterilization. Main advantages of rapid prototyping techniques for surgical guides are that any shape can be built and that patientspecificity of guides does not imply any additional cost. This explains why nowadays, most of the patient-specific surgical guides are built using these techniques. Interestingly, rapid prototyping techniques have also been used to build bone models for improving accuracy and efficiency of surgeries by helping with visualization, understanding, and planning of cases (Bagaria et al. 2011, Brown et al. 2003), as well as by pre-forming implants (Ahn et al. 2006).

Patient-specific surgical guides for orthopedic applications were initially developed for spine surgeries and pelvic osteotomies (Radermacher et al. 1998). Since then, the number of applications has grown drastically, both in academic and industrial environments. One can now find patient-specific guides for various surgical procedures such as total knee replacement (Hafez et al. 2006, Lombardi et al. 2008), total hip replacement (Hananouchi et al. 2009), hip surface replacement (Pattyn et al. 2010, Raaijmaakers et a. 2010, Kunz et al. 2010), intra- and extra- 
articular radius malunions (Leong et al. 2010, Oka et al. 2008), and distal femur osteotomy (Victor et al. 2011), Fig. 6.
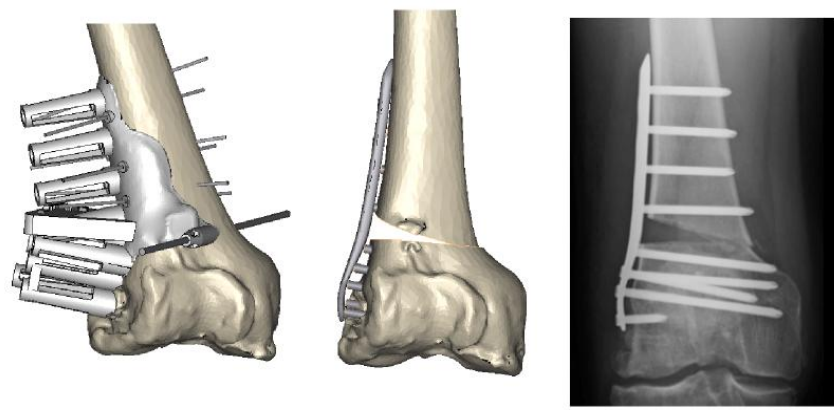

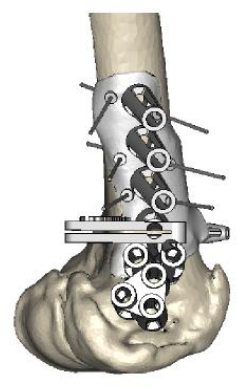

(a)

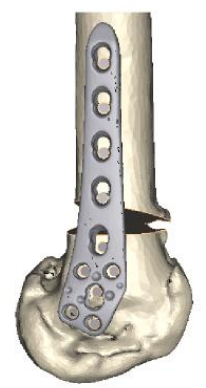

(b)

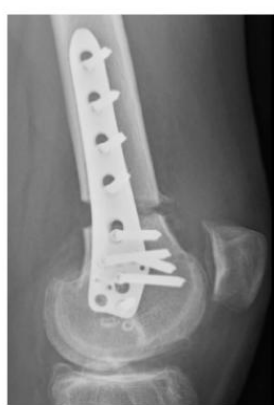

(c)

Fig 6. Illustration of a patient-specific guide for distal femur osteotomy. (a) Guide fit on femur bone model. (b) Planning of surgery. (c) Postoperative X-rays.

For each application, a guide can provide different types of guidance. It can mechanically guide surgical gestures, such as drilling or cutting (Pattyn et al. 2010, Raaijmaakers et al. 2010, Kunz et al. 2010, Leong et al. 2010, Oka et al. 2008, Victor et al. 2011) or indicate anatomical landmarks for positioning standard instruments (Blanz et al. 2004). It can guide the placement of a plate by providing the drilling positions and orientations of plate fixations on preoperative bone (Leong et al. 2010, Victor et al. 2011). It can also visually guide a surgical gesture such as the impaction of a hip implant (Blemker et al. 2007). It can fully replace a standard instrument, becoming for example a patient-specific cutting block for total knee replacement (Hafez et al. 2006). Finally, it can be left in the body, becoming a guiding implant to reposition bone fragments for periacetabular osteotomy (Anaya et al. 2011) or distal radius osteotomy (Oka et al. 2010).

Patient-specific surgical guides were initially developed for increasing the accuracy of procedures (Radermacher et al. 1998). For numerous applications, the goal for using guides is still similar. However, this is not the goal pursued by the use of guides for total knee replacement (TKR), which has by far made the biggest 
progression. This is demonstrated by the routine use of Signature ${ }^{\mathrm{TM}}$ (Biomet), Patient Specific Instruments ${ }^{\circledR}\left(\right.$ Zimmer), OtisKnee ${ }^{\mathrm{TM}}$ (OtisMed), VISIONAIRE ${ }^{\mathrm{TM}}$ (Smith \& Nephew), PROPHECY TM (Wright) or TruMatch ${ }^{\mathrm{TM}}$ (Depuy) for high number of surgeries. The main goal there is to reduce the OR time and to avoid using intramedullar instruments, which also decreases the risk of infection (Hafez et al. 2006). Without the use of a CAS system, a TKR procedure is generally performed in three steps: imaging (e.g. X-rays), planning of the procedure to define the approximate position and size of the implant, and finally surgery itself. During surgery, intramedullar rods and sets of gauges are used to correctly position the cutting blocks on the femur and tibia sides. When using a patient-specific surgical guide such as the Signature ${ }^{\mathrm{TM}}$ guide (Fig.7), the TKR procedure is now performed in five steps. It starts by the imaging of the patient. 3D images (including parts of hip and ankle), such as CT or MRI, are acquired, which is necessary for the subsequent planning and design of the guide. Knee bones, and possibly cartilage, are then segmented from the 3D image. The planning of the TKR consists in defining the position and orientations of the cuts, in addition to the position and the size of the implant. The planning is performed in 3D by calculating the mechanical axes using 3D femur and tibia models. Based on the planned cuts, the position of cutting blocks can be deducted. The surgical guides are then designed such that fixation pins of the cutting blocks are drilled through the guides. During surgery, all the surgical gestures and instruments that are used for deriving the fixation of the cutting blocks can thus be discarded.

Although there is an increasing use of patient-specific surgical guides, interesting challenges remain to make its growth even bigger. Using guides not only requires the acquisition of 3D images, but also a planning performed in 3D. Evolution of the planning, going from 2D on X-rays (as previously often performed) to $3 \mathrm{D}$ on $\mathrm{CT} / \mathrm{MRI}$, will thus be necessary for each procedure. When using surgical guides, going from image acquisition to the surgery itself is lengthened by the 3D segmentation of bones, the 3D planning, the design and production of guides. So far, the lead time for the surgeon to receive the surgical guides remains too long to allow using patient-specific guides for trauma surgeries. Automation of several steps is thus crucial for guides to be used during these surgeries. Finally, most of the patient-specific guides are still not adjustable intraoperatively. Although their use requires thorough planning, which thus implies a lower risk of need for adaptation during surgery, one cannot entirely discard this need. Besides, as explained above, the lead time is still too long to provide new guides rapidly. The development of patient-specific adjustable guides should thus be also envisaged. 


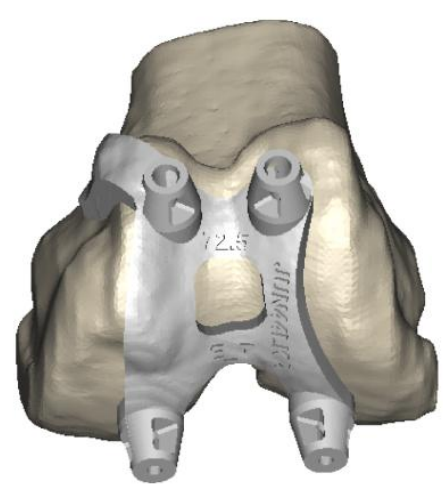

(a)

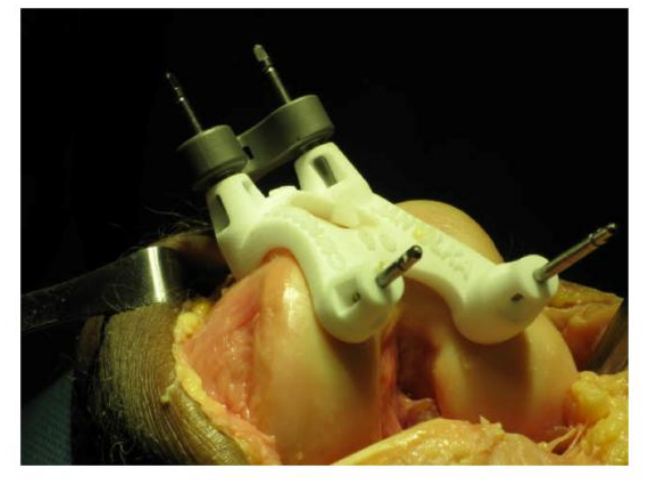

(b)

Fig 7 Illustration of patient-specific Signature ${ }^{\mathrm{TM}}$ femoral guide for total knee replacement. (a) Guide fit on femur bone model. (b) Use of guide during surgery.

\subsection{Computer-assisted surgical planning and navigation}

Surgical planning for osteotomies, tumor resection, implant positioning and deformity correction or bone realignment can easily be performed in 3D before any actual surgery is being performed. Image processing software such as Mimics ${ }^{\circledR}$ (Materialise NV, Heverlee, Belgium) allows for the creation of detailed 3D virtual models from MRI and CT modalities, which can be cut, non-uniformly scaled and oriented in 6 degrees of freedom. In addition and of interest in tumor surgery, data from different imaging modalities can be fused into a single anatomical model (Wong et al. 2010). This is of particular interest in oncology cases for the analysis of tumor extension and its relation with regional anatomy. Fusion of CT and MRI yields hybrid images that combine the key characteristics of each technique, enabling better interpretation and localization of the lesion. The virtual plans can further be exported in DICOM format and used in almost any navigation software (Wong et al. 2010).

Alternatively, and for more complex planning, mesh files such as the STL format can be generated from the patient imaging files and used in a musculoskeletal model package allowing for dynamic simulations and evaluation of "what if" scenarios, to define an optimized surgical plan. Once an optimized virtual plan has been achieved, the resulting data can be re-integrated into the patient's original imaging data set. Again, the resulting data can be exported in DICOM format for further use in computer-assisted surgical navigation (Audenaert et al.2011).

An example of this approach has been used at our orthopedic department for femoroacetabular impingement (FAI). FAI is a mechanical hip disorder defined as 
early and/or repetitive contact between osseous prominences of the acetabulum and/or the proximal femur, potentially resulting in damage to the hip joint in young adults. The prevalence of these lesions - including asymptomatic cases has been reported to be as high as 17-25\%, and multiple studies have shown a significant relationship between radiographic parameters specific of FAI and the development of osteoarthritis of the hip. It has been estimated that osteoarthritis of the hip in Caucasian patients is actually secondary to FAI in $40-70 \%$ of cases. Computer-aided technologies can aid in the early diagnosis and adequate treatment of FAI and thereby prevent or slow down the progression to osteoarthritis. In addition, surgical treatment is technically demanding and involves a steep learning curve. Computer-aided surgery and virtual surgical planning and training can help junior surgeons and surgeons in training to overcome these difficulties and thereby increase the rate of successful surgeries.

Patients are scanned using a 64-slice CT scan (LightSpeed VCT, GE Healthcare, Milwaukee, Wis). A density-based automated segmentation of the femur and pelvis is then performed using the Mimics ${ }^{\circledR}$ image processing software package. Patient-specific STL-format files of the bones are generated and exported for further analysis outside the image analysis software. A custom-designed package is then used for kinematic analysis of the patient's hip joint. The package provides a graphical user interface that allows for morphological evaluation of bony anomalies as well as for simulation of movement and range of motion by collision detection (Audenaert et al. 2010, Audenaert et al. 2011). The necessary amount of bone resection to correct anatomy and restore normal ROM can then be defined. In addition, the effect of possible confounding parameters, e.g. hip joint centre position or soft tissue retraints such as muscles or ligaments impeding motion, can be analyzed (Fig. 8).

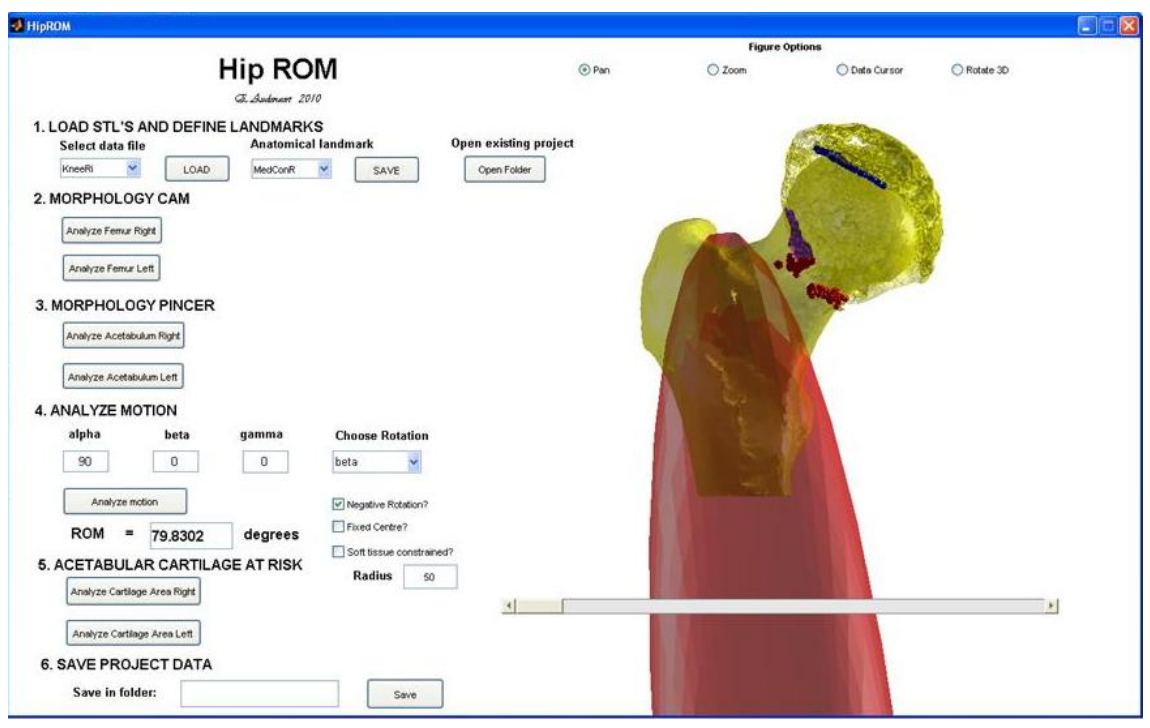


Fig. 8 Patient-specific virtual planning and optimization of treatment.

A virtual plan of the optimized surgical correction to be obtained is exported as STL-format and matched to the original patient imaging data. The virtual plan is then exported in DICOM format for use by the navigation system. In our case the Stryker Orthomap 3D navigation and planning software (Stryker, Mahwah, NJ, USA) is used. The Stryker OrthoMap 3D navigation system requires DICOM image sets as a basis for navigation. Their planning software provides segmentation tools that allow the user to accentuate anatomical structures of interest, such as tumours, bone surfaces and vessels. In our case, a cam and/or pincer lesion is accentuated. The virtual models are matched with the patient's anatomy preoperatively by using a surface-to-surface matching algorithm. Both image-free and image-based navigation protocols can theoretically be used. However, we found significant accuracy problems during the registration process when applying the image-free protocol. The minimal invasive character of the arthroscopic procedure limits access to the relevant bony structures. This impedes accurate surface-tosurface matching. In case of the image-based protocol, intra-operative matching of the patient anatomy was obtained using the Ziehm Vision 3D fluoroscope (Zhiem, Nürnberg, Germany). Finally, a universal tracker can be attached to a calibrated surgical drill and the lesion is resected under computer guidance (Fig. 9). 


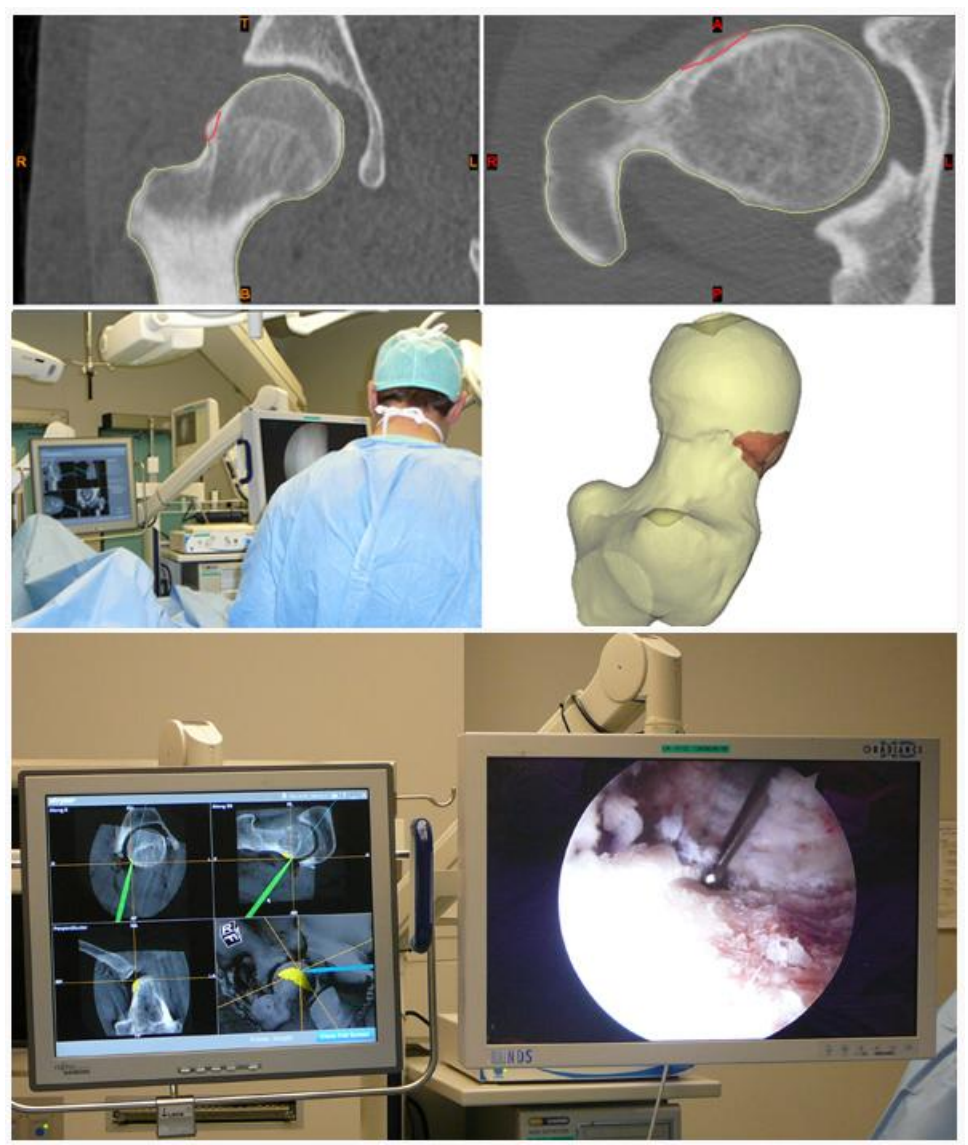

Fig. 9 Clinical case of femoroacetabular impingement. From image to virtual plan and imagebased computer assisted surgery.

\section{References}

Ahn DG, Lee JY, Yang DY (2006) Rapid prototyping and reverse engineering application for orthopedic surgery planning. Journal of mechanical science and technology 20(1): 19-28.

Anaya AM, Vigneron L, Diab M, Burch S (2011) Evaluation of virtual planning, rapid prototyping modeling, and image-guided navigation in periacetabular osteotomy. Proceedings of Computer Assisted Radiology and Surgery 2011.

Audenaert EA, Baelde N, Huysse W, Vigneron L, Pattyn C (2010) Development of a threedimensional detection method of cam deformities in femoroacetabular impingement. Skeletal Radiol. 40:921-7.

Audenaert E, Vigneron L, Pattyn C (2011) A method for three-dimensional evaluation and computer aided treatment of femoroacetabular impingement. Comput Aided Surg 16:143-8.

Bagaria V, Deshpande S, Rasalkar DD, Kuthe A, Paunipagar BK (2011). Use of rapid prototyping and three-dimensional reconstruction modeling in the management of complex fractures. Eur J Radiol. [Epub ahead of print] 
Baldwin MA, Langenderfer JE, Rullkoetter PJ, Laz PJ (2010). Development of subject specific and statistical shape models of the knee using an efficient segmentation and mesh-morphing approach. Comput Methods Programs Biomed 97:232-40.

Barratt DC, Chan CS, Edwards PJ, Penney GP, Slomczykowski M, Carter TJ, et al. (2008) Instantiation and registration of statistical shape models of the femur and pelvis using 3D ultrasound imaging. Med Image Anal 12:358-74.

Behiels G, Maes F, Vandermeulen D, Suetens P (2002) Evaluation of image features and search strategies for segmentation of bone structures in radiographs using active shape models. Med Image Anal 6:47-62.

Benameur S, Mignotte M, Parent S, Labelle H, Skalli W, de Guise J. (2003) 3D/2D registration and segmentation of scoliotic vertebrae using statistical models. Comput Med Imag Graph 27:321-37.

Birnbaum K, Schkommodau E, Decker N, Prescher A, Klapper U, Radermacher K. (2001) Computer-assisted orthopedic surgery with individual templates and comparison to conventional operation method. Spine 26:365-370.

Blanz V, Mehl A, Vetter T, Seidel HP. (2004) A statistical method for robust 3D surface reconstruction from sparse data. In: International symposium on 3D data processing, visualization and transmission. p. 293-300

Blemker SS, Asakawa DS, Gold GE, Delp SL. (2007) Image-based musculoskeletal modeling: applications, advances, and future opportunities. J Magn Reson Imaging. 25(2):441-51.

Bookstein FL (1997) Landmark methods for forms without landmarks: morphometrics of group differences in outline shape. Medical Image Analysis, vol. 1, no. 3, pp. 225-243.

Brown GA, Firoozbakhsh K, DeCoster TA, Reyna JR Jr, Moneim M. (2003) Rapid prototyping the future of trauma surgery? J Bone Joint Surg. 85(4):49-55.

Bryan R, Nair PB, Taylor M. (2009) Use of a statistical model of the whole femur in a large scale, multi-model study of femoral neck fracture risk. J Biomech 42:2171-6.

Cerveri P, Marchente M, Bartels W, Corten K, Simon JP, Manzotti A (2010) Automated method for computing the morphological and clinical parameters of the proximal femur using heuristic modeling techniques. Ann Biomed Eng 38(5):1752-1766.

Cootes, T. F., Taylor, C. J. (2004) Anatomical statistical models and their role in feature extraction. The British Journal of Radiology, 77: S133-S139

Cootes TF, Taylor CJ, Cooper D, Graham J. (1995) Active shape models - their training and application. Computer Vision and Image Understanding 61:38-59.

Cootes TF, Taylor CJ and M. M. Pt. (2004) Statistical models of appearance for computer vision. [Online: http://citeseerx.ist.psu.edu/viewdoc/summary? doi=10.1.1.58.1455.

Delaunay S, Dussault RG, Kaplan PA, Alford BA (1997) Radiographic measurements of dysplastic adult hips. Skeletal Radiol 26(2):75-81

Delp SL, Anderson FC, Arnold AS, Loan P, Habib A, John CT, Guendelman E, Thelen DG. (2007) OpenSim: open-source software to create and analyze dynamic simulations of movement. IEEE Trans Biomed Eng 54:1940-1950.

Delp SL, Loan JP. (1995) A graphics-based software system to develop and analyze models of musculoskeletal structures. Comput Biol Med. 1995 25(1):21-34.

Dryden IL, Mardia K (1998) Statistical Shape Analysis. John Wiley Sons, Chichester.

Ehrhardt J, Handels H, Plötz W, Pöppl SJ (2004) Atlas-based recognition of anatomical structures and landmarks and the automatic computation of orthopedic parameters. Methods Inf Med 43(4):391-397.

Fleute M, Lavallée S (1998) Building a complete surface model from sparse data using statistical shape model. Lect Notes Comput Sci 1496:879-87.

Gomes GT (2011) Automatic feature extraction and statistical shape analysis of the Femur. Submitted to computer methods in biomechanics and biomedical engineering

Grood ES, Suntay WJ (1983) A joint coordinate system for the clinical description of threedimensional motions: application to the knee. J Biomech Eng 105(2):136-144.

Hafez MA, Chelule KL, Seedhom BB, Sherman KP (2006) Computer-assisted total knee arthroplasty using patient-specific templating. Clin Orthop Relat Res. 444:184-192. 
Hananouchi T, Saito M, Koyama T, Hagio K, Murase T, Sugano N, Yoshikawa H. (2009) Tailor-made surgical guide based on rapid prototyping technique for cup insertion in total hip arthroplasty. Int J Med Robot 5(2):164-169.

Hankemeier S, Gosling T, Richter M, Hufner T, Hochhausen C, Krettek C (2006) Computerassisted analysis of lower limb geometry: higher intraobserver reliability compared to conventional method. Comput Aided Surg 11(2):81-86.

Heimann T, Meinzer HP (2009) Statistical shape models for 3D medical image segmentation: a review. Med Image Anal 13:543-63.

Hopkinson N, Hague R, Dickens P (2005) Rapid Manufacturing: An Industrial Revolution for the Digital Age. Wiley.

Jolliffe IT (2002) Principal Component Analysis. 2002; 2nd Edition. Springer.

Kunz M, Rudan JF, Xenoyannis GL, Ellis RE (2010) Computer assisted hip resurfacing using individualized drill templates. The Journal of Arthroplasty 25(4):600-606.

Kurazume R, Nakamura K, Okada T, Sato Y, Sugano N, Koyama T, Iwashita Y, and Hasegawa $\mathrm{T}$ (2009) 3D reconstruction of a femoral shape using a parametric model and two $2 \mathrm{~d}$ fluoroscopic images. Comput. Vis. Image Underst. 113(2): 202-211.

Lamecker H, Seebass M, Hege HC, and Deuflhard P (2004) A 3d statistical shape model of the pelvic bone for segmentation J. M. Fitzpatrick and M. Sonka, Eds., vol. 5370, no. 1. SPIE, 1341-1351.

Leong NL, Buijze GA, Fu EC, Stockmans F, Jupiter JB (2010) Computer-assisted versus noncomputer-assisted preoperative planning of corrective osteotomy for extra-articular distal radius malunions: a randomized controlled trial. BMC Musculoskeletal Disorders 11:282.

Lerner AL, Tamez-Pena JG, Houck JR, Yao J, Harmon HL, Salo AD, Totterman SM (2003) The use of sequential MR image sets for determining tibiofemoral motion: reliability of coordinate systems and accuracy of motion tracking algorithm. J Biomech Eng 125(2):246-253.

Lombardi AV, Berend KR, Adams JB (2008) Patient-specific Approach in Total Knee Arthroplasty. Orthopedics 31(9): 927-930.

Morton NA, Maletsky LP, Pal S, Laz PJ (2007) Effect of variability in anatomical landmark location on knee kinematic description. J Orthop Res 25(9):1221-1230.

Nizard R (2002) Computer assisted surgery for total knee arthroplasty. Acta Orthop Belg 68(3):215-230.

Nofrini L, Slomczykowski M, Iacono F, Marcacci M (2004) Evaluation of accuracy in ankle center location for tibial mechanical axis identification. J Invest Surg 17(1):23-29.

Oka K, Moritomo H, Goto A, et al. (2008) Corrective osteotomy for malunited intra-articular fracture of the distal radius using a custom-made surgical guide based on three-dimensional computer simulation: case report. J Hand Surg Am 33:835-840.

Oka K, Murase T, Moritomo H, Goto A, Sugamoto K, Yoshikawa H. Corrective osteotomy using customized hydroxyapatite implants prepared by preoperative computer simulation. (2010) Int J Med Robot. 6(2):186-193.

Paley D (2002) Normal lower limb alignment and joint orientation. In: Paley D, Herzenberg JE Principles of Deformity Correction. Springer-Verlag, New York.

Pattyn C, De Smedt K, Gelaude F, Clijmans T, Dille J, Geebelen B, Audenaert E (2010) A custom-made guide for femoral component positioning in hip resurfacing arthroplasty: development and validation study. Journal of Biomechanics 43(1).

Raaijmaakers M, Gelaude F, De Smedt K, Clijmans T, Dille J, Mulier M (2010) A custom-made guide-wire positioning device for hip surface replacement arthroplasty: description and first results. BMC Musculoskelet Disord. 11:161.

Radermacher K, Portheine F, Anton M, Zimolong A, Kaspers G,Rau G, Staudte HW (1998) Computer assisted orthopaedic surgery with image-based individual templates. Clin Orthop Relat Res. 354:28-38.

Rajamani KT, Styner MA, Talib H, Zheng G, Nolte LP, Gonzáles Ballester MA (2007) Statistical deformable bone models for robust 3D surface extrapolation from sparse data. Med Image Anal 11:99-109. 
Rueckert D, Sonoda LI, Hayes C, Hill DLG, Leach MO, and Hawkes DJ (1999) Non-rigid registration using free-form deformations: Application to breast mr images. IEEE Transaction on Medical Imaging, 18:712 - 721 .

Schöttle PB, Schmeling A, Rosenstiel N, Weiler A (2007) Radiographic land-marks for femoral tunnel placement in medial patellofemoral ligament reconstruction. Am J Sports Med 35(5):801-804.

Siston RA, Giori NJ, Goodman SB, Delp SL (2007) Surgical navigation for total knee arthroplasty: a perspective. J Biomech 40(4):728-735.

Styner M, Lieberman JA, McClure RK, Weinberger DR, Jones DW, and Gerig G (2005) Morphometric analysis of lateral ventricles in schizophrenia and healthy controls regarding genetic and disease specific factors. Proceedings of the National Academy of Sciences, 102(13):4872-4877.

Stindel E, Briard JL, Merloz P, Plaweski S, Dubrana F, Lefevre C, et al. ( 2002) Bone morphing: 3D morphological data for total knee arthroplasty. Comput Aid Surg 7:156-68.

Subburaj K, Ravi B, Agarwal M (2010) Computer-aided methods for assessing lower limb deformities in orthopaedic surgery planning. Comput Med Imaging Graph 34(4):277-288.

Subburaj K, Ravi B, Agarwal M (2009) Automated identification of anatomical landmarks on 3D bone models reconstructed from CT scan images. Comput Med Imaging Graph 33(5):359-368

Tang TS, Ellis RE (2005) 2D/3D deformable registration using a hybrid atlas. Med Image Comput Assist Interv 8:223-30.

Van Cauter S, De Beule M, Van Haver A, Verdonk P, Verhegghe B (2011) Automated extraction of the femoral anatomical axis for determining the intramedullary rod parameters in total knee arthroplasty. Accepted for publication in International Journal for Numerical Methods in Biomedical Engineering.

Van Sint Jan S, Della Croce U (2005) Identifying the location of human skeletal landmarks: why standardized definitions are necessary-a proposal. Clin Biomech (Bristol, Avon) 20(6):659660.

Van Sint Jan S (2007) Color Atlas of skeletal landmark definitions. Guidelines for reproducible manual and virtual palpations. Churchill Livingstone-Elsevier, Edinburgh.

Victor J, Deprez P, Premanathan A, Keppler L. (2011) Virtual 3d planning and patient specific surgical guides for osteotomies around the knee. Proceedings of Computer Assisted Orthopaedics Surgery 2011

Victor J, Van Doninck D, Labey L, Innocenti B, Parizel PM, Bellemans J (2009) How precise can bony landmarks be determined on a CT scan of the knee? Knee 16(5):358-365.

Wolf A, Digioia AM 3rd, Mor AB, Jaramaz B (2005) Cup alignment error model for total hip arthroplasty. Clin Orthop Relat Res (437):132-13

Wong KC, Kumta SM, Antonio GE, Tse LF (2008) Image fusion for computer-assisted bone tumor surgery. Clin Orthop Relat Res. 466(10):2533-41.

Wong KC, Kumta SM, Leung KS, Ng KW, Ng EW, Lee KS. (2010) Integration of CAD/CAM planning into computer assisted orthopaedic surgery. Comput Aided Surg. 15(4-6):65-74.

Yang, YM., Rueckert, D, Bull, A.M.J (2008) Predicting the shapes of bones at a joint: application to the shoulder. Computer Methods in Biomech. and Biomed. Eng., 11(1):19-30.

Yoon YS, Hodgson AJ, Tonetti J, Masri BA, Duncan CP (2008) Resolving inconsistencies in defining the target orientation for the acetabular cup angles in total hip arthroplasty. Clin Biomech 23(3):253-259.

Yoshino N, Takai S, Ohtsuki Y, Hirasawa Y (2001) Computed tomography measurement of the surgical and clinical transepicondylar axis of the distal femur in osteoarthritic knees. J Arthroplasty 16(4):493-497.

Zheng G, Gollmer S, Schumann S, Dong X, Feilkas T, and Ballester MA G (2008) A 2d/3d correspondence building method for reconstruction of a patient-specific $3 \mathrm{~d}$ bone surface model using point distribution models and calibrated X-ray images. Medical Image Analysis 13(6): 883-899. 
Zheng G, Gonzáles-Ballester MA, Styner M, Nolte LP. (2006) Reconstruction of patient specific $3 \mathrm{~d}$ bone surface from $2 \mathrm{~d}$ calibrated fluoroscopic images and point distribution model. Lect Notes Comput Sci 4190:25-32.

Zheng G, Schumann S (2009) 3D reconstruction of a patient-specific surface model of the proximal femur from calibrated X-ray radiographs: a validation study. Med Phys 36:1155-66.

Ziegler CG, Pietrini SD, Westerhaus BD, Anderson CJ, Wijdicks CA, Johansen S, Engebretsen L, Laprade RF (2010) Arthroscopically Pertinent Landmarks for Tunnel Positioning in Single-Bundle and Double-Bundle Anterior Cruciate Ligament Reconstructions. Am J Sports Med Dec 20. [Epub ahead of print] 